\title{
Vốn xã hội, sự cam kết với tổ chức và chia sẻ tri thức ẩn: Nghiên cứu cho khu vực công tại Thành phố Hồ Chí Minh
}

\section{Social capital, organizational commitment and tacit knowledge sharing: A study on the public sector in Ho Chi Minh City}

\author{
Đoàn Bảo Sơn ${ }^{1}$ \\ ${ }^{1}$ Học viện Hàng không Việt Nam, Việt Nam \\ *Tác giả liên hệ, Email: sondb@vaa.edu.vn
}

THÔNG TIN

DOI: $10.46223 / \mathrm{HCMCOUJS}$.

soci.vi.16.2.1986.2021

Ngày nhận: 07/07/2021

Ngày nhận lại: 21/07/2021

Duyệt đăng: 31/07/2021

Tù khóa:

cam kết với tổ chức; chia sẻ tri thức ẩn; khu vực công; SEM; vốn xã hội

Keywords:

organizational commitment; tacit knowledge sharing; public sector; SEM; social capital

\section{TÓM TÁT}

Nghiên cứu này khám phá các nhân tố làm tiền đề cho hành vi chia sẻ tri thức ẩn trong khu vực công. Dữ liệu được thu thập bằng bảng hỏi và theo phương pháp lấy mẫu thuận tiện. Đối tượng khảo sát là cán bộ, công chức, viên chức đang làm việc tại các cơ quan hành chính nhà nước ở Thành phố Hồ Chí Minh. Bằng sự hỗ trợ của các lãnh đạo, tác giả thu được 477 bảng trả lời từ các đáp viên. Kết quả kiểm định các giả thuyết nghiên cứu bằng mô hình cấu trúc tuyến tính ( $\mathrm{SEM}$ ) cho thấy 03 chiểu kích vốn xã hội cấu trúc, vốn xã hội quan hệ và vốn xã hội tri nhận đều ảnh hưởng tích cực đến sự cam kết với tổ chức. Đến lượt nó, sự cam kết của nhân viên sẽ thúc đẩy hành vi chia sẻ tri thức ẩn. Dựa vào các kết quả này, các hàm ý chính sách thúc đẩy nhân viên chia sẻ tri thức ẩn cũng được tác giả đề xuất trong bài báo.

\section{ABSTRACT}

This paper explores enablers of Tacit Knowledge Sharing (TKS) behavior in the public sector. Surveys were designed by structured questionnaires to collect data using convenience sampling. Respondents are civil servants and officials have been working in state administrative agencies in Ho Chi Minh City. Thanks to leader supports, the author obtained 477 responses. The empirical findings using Structural Equation Modeling (SEM) approach show that three dimensions of structural social capital, relational social capital and cognitive social capital all positively affect Organizational Commitment (OC). In turn, TKS is stimulated by OC. Based on these results, the policies implications of promoting employees to share tacit knowledge are also proposed in this article.

\section{Giới thiệu}

Ngày nay, các tổ chức trong khu vực công là các tổ chức chuyên sâu về tri thức (Rasdi \& Tangaraja, 2020). Tính tiết kiệm, hiệu quả, sáng tạo, đột phá và đổi mới của các dịch vụ công cung cấp phụ thuộc vào chia sẻ tri thức giữa các nhân viên (Manaf, Armstrong, Lawton, \& Harvey, 2018). Tri thức được chia sẻ giữa các nhân viên có thể là ẩn hoặc hiện (Nonaka, 1994). 
Trong đó, tri thức ẩn là nguồn gốc của mọi tri thức tổ chức (Nonaka \& Takeuchi, 1995) và hữu ích hơn trong thực hành quản lý (Bennet \& Bennet, 2008). Nghiên cứu về tri thức ẩn thực sự quan trọng vì nó liên quan đến sự sáng tạo và hành vi thực tế của nhân viên thông qua kinh nghiệm (Wagner \& Sternberg, 1985) và đặc biệt hữu ích trong việc đánh giá những khác biệt về hiệu quả làm việc của nhân viên (Fang \& Zhang, 2014). Sự thành công của các sáng kiến quản lý tri thức có thể phụ thuộc nhiều vào sự sẵn sàng chia sẻ tri thức của mỗi cá nhân (Hislop, 2003). Mặc dù đã có những đề xuất rằng hành vi sẵn sàng chia sẻ tri thức ẩn (TKS) của nhân viên phụ thuộc vào sự cam kết với tổ chức $(\mathrm{OC})$, nhưng có rất ít nghiên cứu liên quan đến chủ đề này (Lin, 2007). Hơn nữa, vốn xã hội (SC) là một tập hợp các nguồn lực xã hội có trong các mối quan hệ giữa các cá nhân (Reed, Srinivasan, \& Doty, 2009) và các nghiên cứu trước về SC đã chứng minh rằng nó có liên quan đến thành công trong sự nghiệp của nhân viên (Seibert, Kraimer, \& Liden, 2001), tạo ra giá trị tổ chức (Tsai \& Ghoshal, 1998) và lợi thế bền vững (Nahapiet \& Ghoshal 1998). Đặc biệt, SC có tác động thúc đẩy OC của nhân viên trong khu vực công (Hsu, Chang, Huang, \& Chiang, 2011; Rasdi \& Tangaraja, 2020). Đối với Việt Nam và vùng kinh tế trọng điểm phía Nam, Thành phố Hồ Chí Minh có vị trí, vai trò rất quan trọng, tạo động lực phát triển kinh tế - xã hội cho các tỉnh trong vùng và cả nước. Do đó, thành phố cần phải có các chính sách hướng đến mục tiêu phát triển bền vững dựa trên kinh tế tri thức, thúc đẩy tăng trưởng xanh, cũng như tập trung xây dựng nguồn nhân lực có đủ trình độ, năng lực phục vụ. Để khuyến khích Cán Bộ, Công Chức, Viên Chức (CBCCVC) gắn bó với khu vực công, thu hút được nhân tài, lao động chuyên môn cao, Thành phố Hồ Chí Minh được chọn là địa phương thí điểm đầu tiên trả thêm thu nhập cho CBCCVC dựa trên kết quả công việc. Để thực thi chính sách này, những nhà quản lý cần dựa vào năng suất và hiệu quả làm việc để đánh giá đầu ra của CBCCVC. Trong bối cảnh khu vực công ở Thành phố Hồ Chí Minh, nghiên cứu này sẽ điều tra các chiều kích của $\mathrm{SC}$ có thể làm tiền đề thúc đẩy $\mathrm{TKS}$ và cũng để giải thích tác động điều tiết của $\mathrm{OC}$ đối với quan hệ giữa $\mathrm{SC}$ và $\mathrm{TKS}$.

Ngoài phần giới thiệu, bài báo này được cấu trúc gồm có các phần như sau: phần 2 là các lý thuyết liên quan và phát triển mô hình nghiên cứu, phần 3 trình bày phương pháp nghiên cứu, phần 4 sẽ phân tích kết quả nghiên cứu, phần 5 thảo luận kết quả và hàm ý chính sách, cuối cùng là kết luận và đề xuất nghiên cứu trong tương lai.

\section{Cơ sở lý thuyết và mô hình nghiên cứu}

\subsection{Các cấu trúc trong mô hình}

\subsubsection{Vốn xã họi (SC - Social Capital)}

Các nghiên cứu về cộng đồng là nơi khởi nguồn của thuật ngữ "vốn xã hội" (Jacobs, 1961, p. 148) và nó được mô tả như một tài sản kết tinh với các mối quan hệ của cá nhân, mạng lưới, cộng đồng hoặc xã hội. Do đó, $\mathrm{SC}$ bao gồm “các mạng lưới xã hội và các chuẩn mực có đi có lại và đáng tin cậy phát sinh từ chúng” (Putnam, 2000, p. 19). SC đề cập đến tổng các nguồn lực tồn tại bên trong hình thành từ mạng lưới các mối quan hệ của các cá nhân (Nahapiet \& Ghoshal, 1998) và tồn tại nhờ vào mối quan hệ gần gũi giữa các cá nhân (Bolino, Turnley, \& Bloodgood, 2002). SC thúc đẩy những cá nhân tham gia thực hiện hành động tập thể hiệu quả hơn trong việc hướng đến các mục tiêu chung của họ thông qua các đặc tính của mạng lưới xã hội, chuẩn mực và sự tin tưởng (Putnam, 1995).

Dựa trên khung lý thuyết do Nahapiet và Ghoshal (1998) đề xuất, nghiên cứu này xem xét SC với ba chiều kích: 1) cấu trúc (Structural SC - SSC), 2) quan hệ (Relational SC - RSC) và 3) tri nhận (Cognitive SC - CSC). Chiều kích SSC đề cập đến mô hình liên kết tổng thể giữa các cá nhân bao gồm các ràng buộc mạng lưới, cấu hình mạng lưới và tổ chức phù hợp. Trong các nghiên cứu về chia sẻ tri thức, SSC được hiểu là các mối quan hệ tương tác xã hội (Hau, Kim, \& 
Lee, 2016). RSC đề cập đến các mối quan hệ cá nhân được hình thành thông qua lịch sử tương tác bao gồm niềm tin, chuẩn mực, sự gắn bó và nghĩa vụ. Trong các khía cạnh của RSC, tác giả tập trung vào niềm tin vì niềm tin giữa các cá nhân là một trong những nhân tố quan trọng nhất thúc đẩy hành vi tìm kiếm tri thức (Mohammed \& Kamalanabhan, 2019). Cuối cùng, CSC là mức độ mà các thành viên của nhóm chia sẻ sự hiểu biết hoặc quan điểm chung. Nahapiet và Ghoshal (1998) liên hệ nó với những tự sự chung và ngôn ngữ chung, trong khi các học giả khác đã bổ sung thêm mục tiêu hoặc tầm nhìn chung và văn hóa chung (Tsai \& Ghoshal, 1998). Trong bối cảnh nghiên cứu hiện tại, $\mathrm{CSC}$ được hiểu là tầm nhìn chung và ngôn ngữ chung.

\subsubsection{Sự cam kết với tổ chức (OC - Organizational Commitment)}

“Cam kết là sức mạnh ràng buộc một cá nhân với một quá trình hành động có liên quan đến một hoặc nhiều mục tiêu” (Meyer \& Herscovitch, 2001, p. 301). Cam kết là động lực ổn định định hướng cho hành vi. Hành vi trọng tâm là để tồn tại trong tổ chức, trong khi hành vi tùy nghi có thể linh hoạt đối với bất kỳ hành vi công dân nào. Những nhân viên có cam kết với tổ chức cũng sẽ có khuynh hướng hoàn thành mục tiêu của tổ chức và tham gia vào các hành vi có liên quan đến tổ chức, chẳng hạn như chia sẻ tri thức (Rasdi \& Tangaraja, 2020). Mô hình $\mathrm{OC}$ (Meyer \& Allen, 1991) có ba chiều kích: tình cảm, lâu dài và quy chuẩn. Cam kết tình cảm dựa trên tư duy được đặc trưng bởi mong muốn tuân theo một quy trình hành động phù hợp với mục tiêu. Cam kết lâu dài dựa trên tư duy được đặc trưng bởi chi phí cảm nhận. Cam kết chuẩn mực dựa trên tư duy được đặc trưng bởi nghĩa vụ được nhận thức (Meyer \& Herscovitch, 2001). Tất cả ba hình thức cam kết này có thể hiện diện đồng thời trong tư duy của nhân viên, do đó $\mathrm{OC}$ là kết hợp của ba trạng thái tâm lý này, chúng đều liên quan đến những hành vi tích cực phù hợp với tổ chức, mà chia sẻ tri thức là một hành vi như thế (Rasdi \& Tangaraja, 2020).

\subsubsection{Chia sẻ tri thức ẩn (TKS - Tacit Knowledge Sharing)}

Nonaka (1994) đã phân loại tri thức của tổ chức bao gồm tri thức hiện và tri thức ẩn. Trong đó, tri thức ẩn là những hiểu biết, trực giác và linh cảm chủ quan, nó bắt nguồn sâu xa từ hành động và kinh nghiệm của một cá nhân, cũng như trong những ý tưởng, giá trị hoặc cảm xúc mà người đó chấp nhận (Nonaka \& Takeuchi, 1995). Tri thức ẩn về cơ bản nằm trong tâm trí của các cá nhân và được thể hiện dưới dạng hành động của con người như thái độ, cam kết và động lực (Nonaka \& Krogh, 2009). Trong việc thực hiện hoạch định chiến lược, tri thức ẩn là nguồn lực quan trọng đối với các nhà quản lý và nhân viên (Brockmann \& Anthony, 1998), làm tăng chất lượng hoàn thành công việc, cũng như phục vụ khách hàng tốt hơn và nâng cao hiệu quả làm việc (Herrgard, 2000). Theo Nonaka và Toyama (2003), tri thức được tạo ra từ xã hội hóa (socialization), đó là quá trình chuyển đổi tri thức ẩn mới thông qua chia sẻ kinh nghiệm nhờ tương tác xã hội hàng ngày và hầu hết có thể nhận được thông qua kinh nghiệm trực tiếp. Do đó, việc chia sẻ tri thức ẩn (TKS) - có thể là kết quả của các cuộc họp chính thức như hội nghị và chương trình đào tạo, nhưng phần lớn hành vi TKS diễn ra thông qua mạng lưới xã hội và sự tương tác giữa các nhân viên (Holste \& Fields, 2010). Ngoài ra, mấu chốt của TKS nằm ở năng lực và sự sẵn sàng của các cá nhân trong việc chia sẻ những gì họ biết (đóng góp tri thức) và sử dụng những gì họ học được (thu thập tri thức) (Holste \& Fields, 2010).

\subsection{Mô hình nghiên cúu}

\subsubsection{Mối quan hệ giũa $S C$ và $O C$}

Chiều kích SSC tập trung vào các tương tác xã hội giữa các nhân viên, tức là với ai và với tần suất họ thực hiện các hành động giao tiếp để chia sẻ thông tin (Hsu et al., 2011). Một mạng lưới các mối quan hệ cho biết thông tin về những sự kiện đang xảy ra trong tổ chức, khả năng truy cập các cấu trúc quyền lực, hỗ trợ tinh thần và tình bạn. Điều này sẽ khuyến khích các cá nhân tham gia vào các công việc của tổ chức và gắn bó với tổ chức, do đó quyết định đến mức 
độ OC (Bozionelos, 2008). Hơn nữa, nhờ mạng lưới các mối quan hệ mà nhân viên thiết lập trong tổ chức, họ có nhiều khả năng cảm nhận được việc rời bỏ tổ chức của mình về khía cạnh các chi phí liên quan vì khi chuyển sang một tổ chức khác sẽ đòi hỏi nỗ lực và thời gian để xây dựng lại một mạng lưới như vậy và sẽ kéo theo sự không chắc chắn về việc liệu nỗ lực này có thành công hay không (Bozionelos, 2008). Do đó, các nguồn lực mạng lưới càng mạnh thì sự cam kết lâu dài với tổ chức của nhân viên càng tăng. Dựa vào các lập luận này, giả thuyết $\mathrm{H} 1$ được đề xuất như sau:

\section{H1: SSC có ảnh hưởng tích cục đến OC}

Niềm tin là nhân tố quan trọng trong cấu trúc văn hóa tổ chức, hiệu quả và sự cam kết của nhân viên với tổ chức (Laschinger, Finegan, \& Shamian, 2002). Niềm tin tổ chức có hai chiều kích là nhận thức và cảm xúc (Song, Kim, \& Kolb, 2009). Niềm tin nhận thức được xác định bởi văn hóa (Tekingündüz, Top, Tengilimoğlu, \& Karabulut, 2017), còn niềm tin cảm xúc liên quan đến các mối quan hệ cá nhân, tình cảm cũng như các giao tiếp chất lượng cao và góp phần tạo nên mối liên kết tình cảm bền chặt hơn (Lievens \& Corte, 2008), do đó hỗ trợ OC về tình cảm. Trong môi trường làm việc, $\mathrm{OC}$ còn thể hiện rằng nhân viên cần ở lại tổ chức trước khi họ tìm kiếm được một công việc tốt hơn hoặc phù hợp hơn cho mình (Allen \& Meyer, 1990). Mối quan hệ tin cậy có đi có lại giữa những nhân viên có thể tạo ra ý thức về nghĩa vụ đạo đức để tiếp tục gắn bó với tổ chức dựa trên sự chia sẻ các giá trị và niềm tin (Curado \& Vieira, 2019). Do đó, tác giả đề xuất mối quan hệ giữa $\mathrm{RSC}$ và $\mathrm{OC}$ bằng giả thuyết $\mathrm{H} 2$ dưới đây:

\section{H2: RSC có ảnh hưởng tích cực đến $O C$}

Các mục tiêu và nguyện vọng chung của các thành viên trong tổ chức được hiểu là tầm nhìn chung (Tsai \& Ghoshal, 1998) và chiều kích này giúp cho các thành viên nhận biết được chân lý của vấn đề (Watson \& Papamarcos, 2002). Tầm nhìn chung có thể ngăn chặn những hiểu lầm trong giao tiếp giữa các cá nhân và thúc đẩy tự do trao đổi ý tưởng và nguồn lực (Tsai \& Ghoshal, 1998), từ đó thúc đẩy nhân viên có sự gắn bó với các tổ chức (Hsu et al., 2011). Bản chất của tầm nhìn là giá trị và mục tiêu, khi con người có mục tiêu và giá trị, cũng có nghĩa là họ có nhu cầu cơ bản về các định hướng giá trị và ý thức về mục tiêu làm cho cho cuộc sống và công việc có nhiều ý nghĩa hơn. Vì vậy, tầm nhìn giúp mọi người cam kết và cũng đóng vai trò là trọng tâm (mục tiêu) và hướng dẫn hành vi (giá trị) để hướng tới mục tiêu của tổ chức (Coetsee, 1999). Khi nhân viên hiểu và cho rằng tổ chức có sứ mệnh, tầm nhìn và giá trị là phù hợp thì họ có nhiều khả năng sẽ điều chỉnh các giá trị và mục tiêu của mình hướng đến các giá trị và mục tiêu của tổ chức. Điều này có nghĩa là nhân viên sẽ cảm thấy gắn bó với tổ chức hơn (Chai, Hwang, \& Joo, 2017). Với các lập luận này, tác giả kỳ vọng OC được tăng cường cùng với CSC bằng giả thuyết như sau:

\section{H3: CSC có ảnh hương tích cực đến OC}

\subsubsection{OC là tiền đề của TKS}

TKS được tăng cường khi nhân viên có mức $\mathrm{OC}$ tương đối cao vì bản chất tự nguyện của nó dựa trên sự sẵn sàng truyền đạt tri thức (Nonaka, 1994). OC là mức độ gắn kết và tham gia của cá nhân với các giá trị và mục tiêu của tổ chức (Borges \& Mote, 2016). Nhân viên có OC sẵn sàng nỗ lực hơn nữa cho tổ chức, đồng thời họ cũng chăm chỉ làm việc hơn để đạt được mục tiêu và đáp ứng kỳ vọng của tổ chức (Meyer \& Allen, 1991). Do đó, trong tổ chức có văn hóa hướng tập thể và hỗ trợ, mức độ $\mathrm{OC}$ càng cao thì nhân viên sẽ càng có nhiều khả năng tham gia vào các hành vi TKS. Những nhân viên tận tâm, có mức độ ổn định cảm xúc có thể TKS khi họ cam kết với tổ chức nhiều hơn (Borges, Bernardi, \& Petrin, 2019). Những nhân viên có cảm giác gắn bó 
tình cảm với tổ chức, sẽ có khả năng chia sẻ tri thức nếu họ nhận ra rằng hành vi đó được đánh giá cao và mang lại lợi ích thực sự cho tổ chức (Hooff \& Weenen, 2004). Nhân viên có cam kết mạnh mẽ, sẽ thường mang lại sự tập trung cho tư cách thành viên tổ chức của họ cũng như tăng cường sự tương tác với các đồng nghiệp (O’Reilly \& Chatman, 1986). Do đó, sự gắn kết này có thể tăng cường cam kết để thúc đẩy hành vi TKS của họ (Lin, 2007). Dựa vào các lý thuyết hiện tại, nghiên cứu này phát biểu giả thuyết $\mathrm{H} 4$ như sau:

H4: OC có tác động tích cực đến TKS

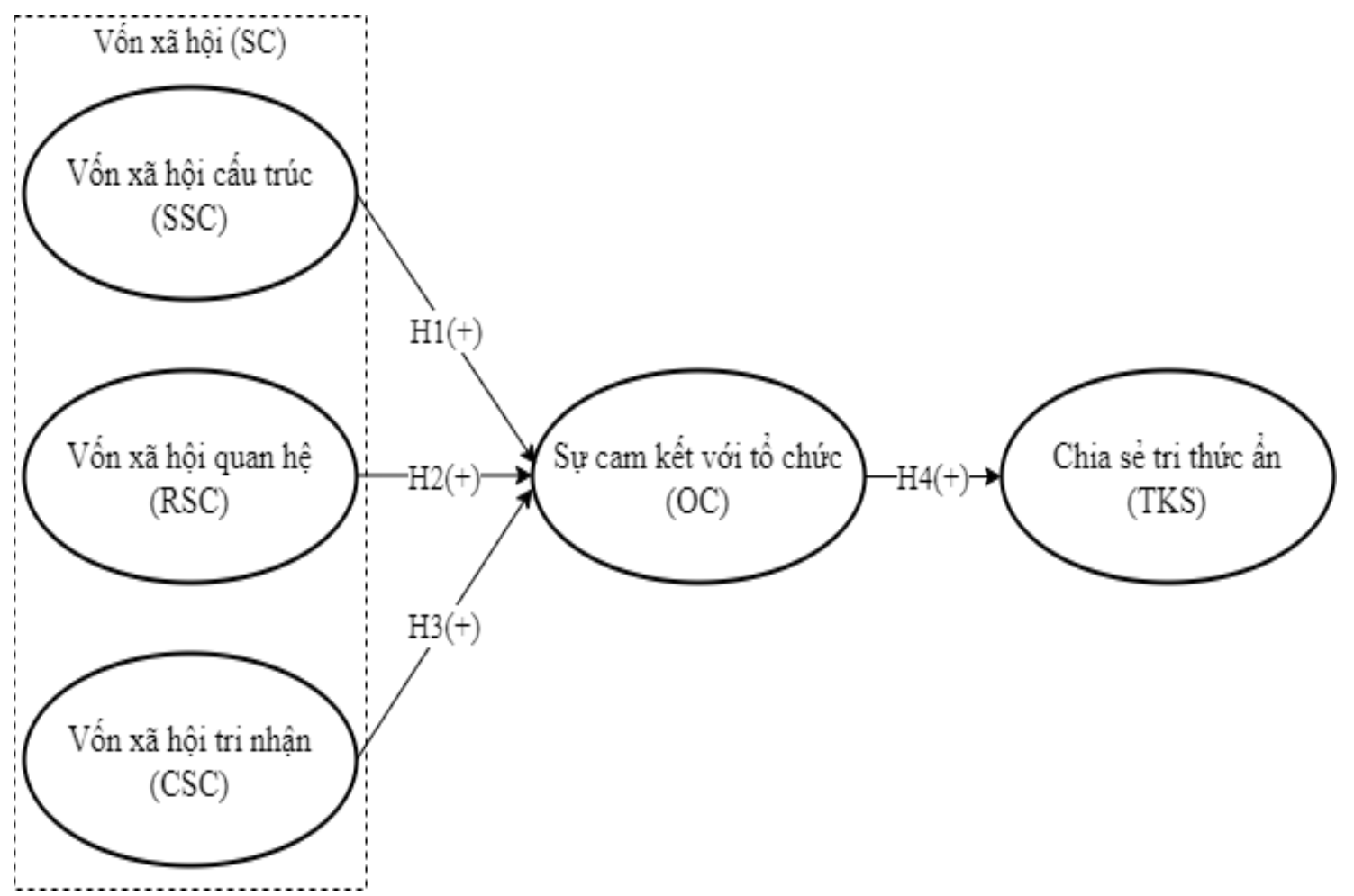

Hình 1. Mô hình nghiên cứu đề xuất

Nguồn: Tác giả

\section{Phương pháp nghiên cứu}

\subsection{Dũ liệu và mẫu nghiên cứu}

Bằng cách sử dụng bảng câu hỏi được cấu trúc theo mục tiêu, tác giả đã phát phiếu khảo sát trực tiếp và trực tuyến qua thư điện tử đến các đáp viên để thu thập dữ liệu trong khoảng thời gian một tháng (03/2021). Phương pháp chọn mẫu thuận tiện là phù hợp trong bối cảnh nghiên cứu này, với đáp viên là $\mathrm{CBCCVC}$ đang công tác tại các sở, ban, ngành ở Thành phố Hồ Chí Minh. Tác giả chọn mẫu là những $\mathrm{CBCCVC}$ có kinh nghiệm công tác tại cơ quan trên 05 năm vì những người mới sẽ không có đủ tri thức ẩn để chia sẻ (Rasdi \& Tangaraja, 2020). Để đạt được độ tin cậy cần thiết, nghiên cứu này phân phối 600 bảng câu hỏi đến các đáp viên. Sau khi loại bỏ các phiếu trả lời không đầy đủ và dị biệt, tác giả thu được 477 mẫu dùng để phân tích dữ liệu, đáp ứng khuyến nghị của Hair, Black, Babin, và Anderson (2014) về cỡ mẫu tối thiểu bằng 200 là phù hợp cho một mô hình đường dẫn phức tạp. Bảng 1 báo cáo dữ liệu nhân khẩu học của những đáp viên được khảo sát. 


\section{Bảng 1}

Bảng mô tả mẫu nghiên cứu

\begin{tabular}{|l|c|c|}
\hline \multicolumn{1}{|c|}{ Đặc điểm nhân khẩu học } & Số lượng & Tỉ lệ phần trăm (\%) \\
\hline Giới tính: & & \\
- Nữ & 226 & 47.4 \\
- Nam & 251 & 52.6 \\
\hline Tuổi: & & 5.5 \\
- Dưới 35 & 26 & 34.4 \\
- 36 - 40 & 164 & 40.6 \\
- 41 - 45 & 194 & 13.6 \\
- 46 - 50 & 65 & 5.9 \\
- Trên 50 & 28 & \\
\hline Trình độ học vấn: & & 0.8 \\
- Trung cấp & 4 & 4.6 \\
- Cao đẳng & 22 & 9.3 \\
- Đại học & 44 & 65.6 \\
- Thạc sĩ & 313 & 19.7 \\
- Khác & 94 & 21.8 \\
\hline Kinh nghiệm công tác (năm): & 147 & 14.3 \\
- 05 - 10 & 68 & 8.6 \\
- 11 - 15 & 41 & 24.5 \\
- 16 - 20 & 117 & \\
- 21 - 25 & & \\
- Trên 25 & & \\
\hline Số mẫu N 477 & & \\
\hline & & \\
\hline
\end{tabular}

Nguồn: Tác giả

\subsection{Thang do}

Nghiên cứu này đo lường các biến quan sát bằng thang đo Likert 5 mức độ, trong đó "1" là "hoàn toàn không đồng ý" và " 5 " là "hoàn toàn đồng ý". Các thang đo được phát triển dựa theo các nghiên cứu trước với những sửa đổi nhỏ để phù hợp với bối cảnh hiện tại. $\mathrm{SC}$ gồm 03 chiều kích là $\mathrm{SSC}, \mathrm{RSC}$ và $\mathrm{CSC}$ được đo lường bằng 12 biến. Trong đó, $\mathrm{SSC}$ đề cập đến sức mạnh của các mối tương tác mạng lưới giữa nhân viên với các đồng nghiệp, gồm có 04 biến (SSC1 đến SSC4) tham khảo từ Chiu, Hsu, và Wang (2006). RSC dùng để đánh giá niềm tin giữa các nhân viên trong tổ chức, bao gồm 04 biến (RSC1 đến RSC4) dựa theo Mohammed và Kamalanabhan (2019). CSC đề cập đến tầm nhìn chung của các nhân viên được đo bằng 04 biến (CSC1 đến CSC4) tham khảo từ Tsai và Ghoshal (1998). Trong khi đó, 05 biến trong thang đo của $\mathrm{OC}$ (OC1 đến OC5) tham khảo từ Lin (2007). Cuối cùng, thang đo TKS gồm 05 biến quan sát (TKS1 đến TKS5) được phát triển từ Lin (2007) và Wang và Wang (2012). Tất cả các thang đo này được trình bày trong Bảng 2 . 


\section{Bảng 2}

Độ tin cậy của các thang đo

\begin{tabular}{|c|c|c|c|c|c|}
\hline & Các cấu trúc và thang đo & Hệ số tải & Cronbach's & CR & AVE \\
\hline 1. Vốn & xã hội cấu trúc (SSC) & & 0.825 & 0.828 & 0.548 \\
\hline SSC1 & $\begin{array}{l}\text { Duy trì các mối quan hệ thân } \\
\text { mật gần gũi với đồng nghiệp }\end{array}$ & 0.628 & & & \\
\hline $\mathrm{SSC} 2$ & $\begin{array}{l}\text { Dành thời gian đáng kể để } \\
\text { tương tác với đồng nghiệp }\end{array}$ & 0.796 & & & \\
\hline $\operatorname{SSC} 3$ & $\begin{array}{l}\text { Hiểu biết đồng nghiệp ở mức độ } \\
\text { cá nhân }\end{array}$ & 0.798 & & & \\
\hline SSC4 & $\begin{array}{l}\text { Thường xuyên giao tiếp với } \\
\text { dồng nghiệp }\end{array}$ & 0.727 & & & \\
\hline 2. Vốn & ã hội quan hệ (RSC) & & 0.850 & 0.852 & 0.590 \\
\hline $\mathrm{RSC1}$ & $\begin{array}{l}\text { Niềm tin với năng lực thực hiện } \\
\text { nhiệm vụ của đồng nghiệp }\end{array}$ & 0.708 & & & \\
\hline $\mathrm{RSC} 2$ & $\begin{array}{l}\text { Niềm tin rằng đồng nghiệp thực } \\
\text { hiện thành công nhiệm vụ của } \\
\text { mình }\end{array}$ & 0.777 & & & \\
\hline $\mathrm{RSC} 3$ & $\begin{array}{l}\text { Tự tin về kỹ năng của các đồng } \\
\text { nghiệp }\end{array}$ & 0.817 & & & \\
\hline $\mathrm{RSC} 4$ & $\begin{array}{l}\text { Sự quan tâm của đồng nghiệp } \\
\text { đến những gì xảy ra với mình }\end{array}$ & 0.768 & & & \\
\hline 3. Vốn & ã hội tri nhận (CSC) & & 0.864 & 0.865 & 0.616 \\
\hline $\mathrm{CSC} 1$ & $\begin{array}{l}\text { Luôn đồng ý về vấn đề quan } \\
\text { trọng trong công việc }\end{array}$ & 0.799 & & & \\
\hline $\mathrm{CSC} 2$ & $\begin{array}{l}\text { Luôn có chung tham vọng và } \\
\text { tầm nhìn trong công việc }\end{array}$ & 0.724 & & & \\
\hline $\mathrm{CSC} 3$ & $\begin{array}{l}\text { Luôn nhiệt tình theo đuổi mục } \\
\text { tiêu chung và sứ mệnh của toàn } \\
\text { tổ chức }\end{array}$ & 0.826 & & & \\
\hline $\mathrm{CSC} 4$ & $\begin{array}{l}\text { Luôn tạo cơ hội để giao lưu cá } \\
\text { nhân }\end{array}$ & 0.788 & & & \\
\hline 4. Sự ca & m kết với tổ chức (OC) & & 0.867 & 0.868 & 0.569 \\
\hline $\mathrm{OC} 1$ & $\begin{array}{l}\text { Sẵn sàng nỗ lực nhiều hơn } \\
\text { mong đợi để mang đến sự thành } \\
\text { công cho cơ quan }\end{array}$ & 0.771 & & & \\
\hline
\end{tabular}




\begin{tabular}{|c|c|c|c|c|c|}
\hline \multicolumn{2}{|r|}{ Các cấu trúc và thang đo } & \multirow{2}{*}{$\begin{array}{c}\text { Hệ số tải } \\
0.767\end{array}$} & \multirow{2}{*}{$\begin{array}{c}\text { Cronbach's } \\
\text { Alpha }\end{array}$} & \multirow{2}{*}{$\mathbf{C R}$} & \multirow{2}{*}{ AVE } \\
\hline OC2 & $\begin{array}{l}\text { Quan tâm đến số phận của cơ } \\
\text { quan }\end{array}$ & & & & \\
\hline OC3 & $\begin{array}{l}\text { Vô cùng hân hoan khi chọn cơ } \\
\text { quan này để làm việc }\end{array}$ & 0.776 & & & \\
\hline OC4 & $\begin{array}{l}\text { Cho rằng cơ quan này rất tuyệt } \\
\text { vời để làm việc }\end{array}$ & 0.722 & & & \\
\hline OC5 & $\begin{array}{l}\text { Tự hào là nhân viên của cơ } \\
\text { quan này }\end{array}$ & 0.735 & & & \\
\hline \multicolumn{2}{|c|}{ 5. Chia sẻ tri thức ẩn (TKS) } & & 0.893 & 0.893 & 0.627 \\
\hline TKS1 & $\begin{array}{l}\text { Chia sẻ kinh nghiệm công việc } \\
\text { của mình với đồng nghiệp }\end{array}$ & 0.739 & & & \\
\hline TKS2 & $\begin{array}{l}\text { Chia sẻ tri thức chuyên môn } \\
\text { theo yêu cầu của đồng nghiệp }\end{array}$ & 0.751 & & & \\
\hline TKS3 & $\begin{array}{l}\text { Chia sẻ ý tưởng của mình về } \\
\text { công việc với đồng nghiệp }\end{array}$ & 0.774 & & & \\
\hline TKS4 & $\begin{array}{l}\text { Chia sẻ bí quyết trong công việc } \\
\text { với đồng nghiệp }\end{array}$ & 0.841 & & & \\
\hline TKS5 & $\begin{array}{l}\text { Chia sẻ những thất bại trong } \\
\text { quá khứ khi cảm thấy cần thiết }\end{array}$ & 0.846 & & & \\
\hline
\end{tabular}

Nguồn: Tác giả

Bảng 2 cho thấy hệ số tải tiêu chuẩn của các thang đo đều trên mức ngưỡng 0.5 và có ý nghĩa thống kê (Anderson \& Gerbing, 1988; Hair et al., 2014). Ngoài ra, hệ số Cronbach's Alpha của tất cả các cấu trúc có giá trị nhỏ nhất là 0.825 ( $\mathrm{SSC}$ ) nên độ tin cậy thang đo là rất tốt (Kline, 2015).

\section{Kết quả nghiên cứu}

\subsection{Mô hình đo lưòng}

Để đánh giá tính giá trị của mô hình đo lường, nghiên cứu này sẽ kiểm định tính giá trị hội tụ và tính giá trị phân biệt (Anderson \& Gerbing, 1988).

Các kết quả phân tích tính giá trị (Bảng 3) cho thấy độ tin cậy tổng hợp (CR) của các cấu trúc nằm trong khoảng từ 0.828 (SSC) đến 0.893 (TKS) đều trên mức ngưỡng 0.7 và phương sai trích trung bình (AVE) của tất cả các cấu trúc đều trên mức cắt 0.5 (từ 0.548 đến 0.627 ) nên mô hình đo lường được chấp nhận về tính giá trị hội tụ (Hair et al., 2014). Hơn nữa, mỗi cấu trúc đều có căn bậc hai của AVE lớn hơn mối tương quan giữa nó và bất kỳ cấu trúc còn lại (Fornell \& Lacker, 1981) và giá trị AVE của mỗi cấu trúc lớn hơn bình phương hệ số tương quan lớn nhất (MSV) (Hair et al., 2014) nên mô hình đảm bảo tính giá trị phân biệt. Vì vậy, tính hội tụ và phân biệt của mô hình đo lường đã được kiểm định. 


\section{Bảng 3}

Tính giá trị của mô hình đo lường

\begin{tabular}{|l|c|c|c|c|c|c|c|c|c|}
\hline & CR & AVE & MSV & $\begin{array}{c}\text { Căn } \\
\text { bậc hai } \\
\text { của } \\
\text { AVE }\end{array}$ & TKS & OC & SSC & RSC & CSC \\
\hline TKS & 0.893 & 0.627 & 0.470 & 0.792 & 1 & & & & \\
\hline OC & 0.868 & 0.569 & 0.482 & 0.754 & $0.685^{* * *}$ & 1 & & & \\
\hline SSC & 0.828 & 0.548 & 0.387 & 0.740 & $0.512^{* * *}$ & $0.425^{* * *}$ & 1 & & \\
\hline RSC & 0.852 & 0.590 & 0.482 & 0.768 & $0.642^{* * *}$ & $0.694 * * *$ & $0.426^{* * *}$ & 1 & \\
\hline CSC & 0.865 & 0.616 & 0.387 & 0.785 & $0.514^{* * *}$ & $0.421^{* * *}$ & $0.622^{* * *}$ & $0.421^{* * *}$ & 1 \\
\hline
\end{tabular}

Nguồn: Tác giả

Sự phù hợp của mô hình đo lường được kiểm định bằng các chỉ số thống kê như sau: $\mathrm{CMIN} / \mathrm{df}=2.494<3$, với $\mathrm{p}$-value $=0.000 \leq 0.05$ (Hair et al., 2014). Cùng với các thống kê CFI $=0.948>0.92 ; \mathrm{TLI}=0.940>0.92 ; \mathrm{SRMR}=0.0381<0.08$ và $\mathrm{RMSEA}=0.056<0.07$, đều thỏa mãn yêu cầu tối thiểu cho sự phù hợp của mô hình (Hair et al., 2014). Do đó, có thể kết luận rằng mô hình đo lường là phù hợp tuyệt vời.

\subsection{Mô hình cấu trúc}

Kết quả phân tích sự phù hợp của mô hình đường dẫn cho thấy chỉ số CMIN/df $=2.767<$ 3 , p-value $=0.000 \leq 0.05$. Các chỉ số thống kê khác như CFI $=0.938>0.92$, TLI $=0.929>0.92$, SRMR $=0.0622<0.08$, RMSEA $=0.061<0.07$ cũng hoàn toàn phù hợp tốt (Hair et al., 2014). Điều đó chứng tỏ rằng mức độ phù hợp của mô hình cấu trúc có thể chấp nhận được.

\section{Bảng 4}

Kết quả kiểm định quan hệ đường dẫn

\begin{tabular}{|c|c|c|c|c|c|}
\hline \multicolumn{2}{|c|}{ Giả thuyết } & Kỳ vọng & $\begin{array}{c}\text { Ước lượng } \\
\text { chuẩn hóa }\end{array}$ & k-value & Kết luận \\
\hline H1 & $\mathrm{SSC} \rightarrow \mathrm{OC}$ & + & 0.123 & $\begin{array}{c}0.035 \\
(\mathrm{p}<0.05)\end{array}$ & Chấp nhận \\
\hline $\mathrm{H} 2$ & $\mathrm{RSC} \rightarrow \mathrm{OC}$ & + & 0.619 & $\begin{array}{c}* * * \\
(\mathrm{p}<0.001)\end{array}$ & Chấp nhận \\
\hline $\mathrm{H} 3$ & $\mathrm{CSC} \rightarrow \mathrm{OC}$ & + & 0.124 & $\begin{array}{c}0.029 \\
(\mathrm{p}<0.05)\end{array}$ & Chấp nhận \\
\hline $\mathrm{H} 4$ & $\mathrm{OC} \rightarrow \mathrm{TKS}$ & + & 0.727 & $\begin{array}{c}* * * \\
(\mathrm{p}<0.001)\end{array}$ & Chấp nhận \\
\hline
\end{tabular}

Nguồn: Tác giả

Tiếp theo, việc giải thích các tác động đối với các biến phụ thuộc riêng lẻ được thực hiện bằng cách kiểm tra các hệ số ước tính (trọng số) cho mỗi biến. Kết quả phân tích các đường dẫn của mô hình SEM (Bảng 4) chứng minh tất cả các mối quan hệ hồi quy đa biến H1, H2, H3, H4 đều được chấp nhận và có ý nghĩa về mặt thống kê: $\mathrm{p}<0.05$ (đối với $\mathrm{H} 1$ và $\mathrm{H} 3$ ) và $\mathrm{p}<0.001$ (H2 và $\mathrm{H} 4)$. 


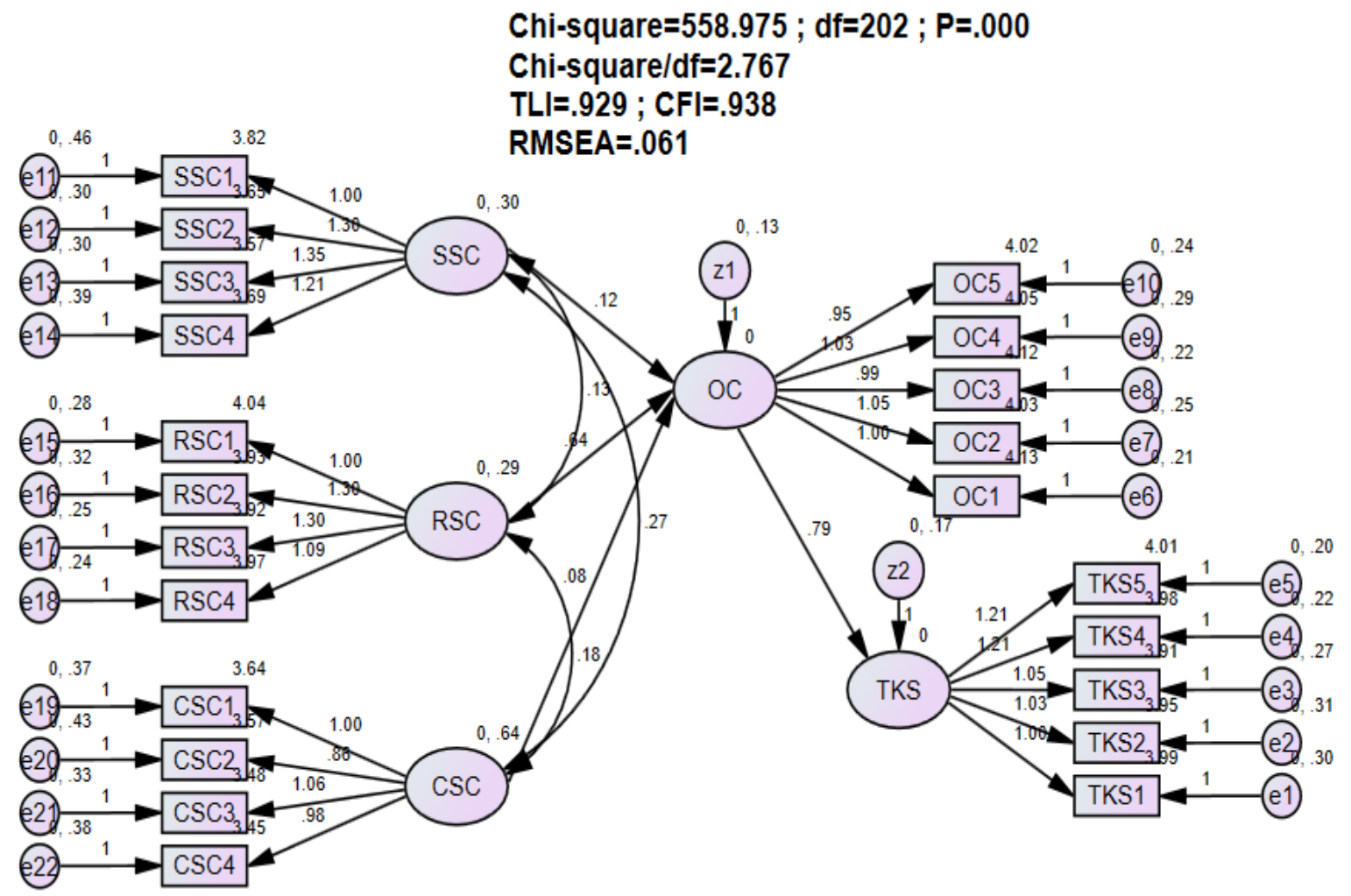

Hình 2. Mô hình SEM

Nguồn: Tác giả

\section{Thảo luận kết quả và hàm ý chính sách}

\subsection{Thảo luận kết quả}

Bài báo này xác định các nhân tố thúc đẩy $\mathrm{OC}$ của nhân viên với tổ chức trong khu vực công và việc cam kết này sẽ mang lại lợi thế gì đối với tri thức ẩn của tổ chức. Tác giả xem xét các chiều kích của $\mathrm{SC}$ là $\mathrm{SSC}, \mathrm{RSC}$ và $\mathrm{CSC}$ như là tiền đề của $\mathrm{OC}$ và kết quả là $\mathrm{TKS}$. Kết quả phân tích hồi quy đa biến trong mô hình cấu trúc chứng minh tất cả mối tương quan giữa các chiều kích của $\mathrm{SC}$ với $\mathrm{OC}$ và $\mathrm{OC}$ với $\mathrm{TKS}$ đều được chấp nhận.

Trước hết, nghiên cứu đã chứng minh các chiều kích SSC, RSC và CSC của $\mathrm{SC}$ đều có tác động cùng chiều đến $\mathrm{OC}$, trong đó nhân tố có tác động mạnh nhất là RSC với ước lượng hồi quy chuẩn hóa là 0.619 ở mức ý nghĩa thống kê $\mathrm{p}<0.001$. Bằng chứng thực nghiệm này được hỗ trợ bởi các lý thuyết hiện tại và các kết quả trước về chủ đề này (Hsu et al., 2011; Rasdi \& Tangaraja, 2020). Mối quan hệ được coi là tài sản của những người tham gia vào hoạt động cộng đồng (Baker, 1990) và $\mathrm{SC}$ thường được gắn vào các mối quan hệ giữa các thực thể (Coleman, 1988). SC được kết nối với sự gắn bó mà nhân viên cam kết với tổ chức (Hsu et al., 2011). Các nghiên cứu trước lập luận rằng $\mathrm{SC}$ có vai trò rất cần thiết đối với $\mathrm{OC}$ và $\mathrm{OC}$ thể hiện sự gắn bó tâm lý của mỗi nhân viên với tổ chức (Gormley \& Kennerly, 2010). Meyer và Herscovitch (2001) gợi ý rằng quá trình xã hội hóa trong tổ chức sẽ hình thành các quy tắc nội bộ và có ảnh hưởng đến mức độ $\mathrm{OC}$ của cá nhân đối với cơ quan của họ. Niềm tin tương hỗ và niềm tin tổ chức cùng với mối tương tác giữa các nhân viên là một trong các yếu tố quy chuẩn nội bộ thường do các quá trình xã hội hóa tổ chức tạo ra (Rasdi \& Tangaraja, 2020). Do đó, phát hiện về tác động mạnh hơn của RSC đối với $\mathrm{OC}$ của bài báo này phù hợp với các thực nghiệm trước đây (Nambudiri, 2012; Rasdi \& Tangaraja, 2020) đều nhấn mạnh rằng niềm tin tạo ra ràng buộc tâm lý giữa nhân viên với các tổ chức khu vực công. 
Nghiên cứu hiện tại còn phát hiện ra $\mathrm{OC}$ thúc đẩy TKS để làm giàu tri thức tổ chức. Kết quả này phù hợp với lý thuyết và các nghiên cứu thực nghiệm của các học giả trước. Sức mạnh của sự tham gia và gắn bó với một tổ chức cụ thể được coi là mức độ $\mathrm{OC}$ của nhân viên (Porter, Steers, Mowday, \& Boulian, 1974). OC có ảnh hưởng tích cực đến việc các cá nhân sẵn sàng cam kết nỗ lực hơn nữa cho thực hiện nhiệm vụ tại nơi làm việc của họ (Meyer \& Allen, 1997), nên nó sẽ làm tăng cường sự sẵn sàng cho và nhận tri thức (Hooff \& Weenen, 2004), đặc biệt là tri thức ẩn với đặc tính rất khó chia sẻ liên quan đến nhận thức, ngôn ngữ, thời gian, giá trị và khoảng cách (Herrgard, 2000). Ngoài ra, trong bối cảnh các tổ chức công, Lin (2007) cho thấy sự hình thành $\mathrm{TKS}$ bằng cách coi $\mathrm{OC}$ và sự tin tưởng vào đồng nghiệp là hai biến trung gian quan trọng, cũng như Rasdi và Tangaraja (2020) chứng minh cam kết tình cảm và ý thức nghĩa vụ đối với tổ chức là các nhân tố quan trọng để đảm bảo chia sẻ tri thức giữa các nhân viên.

\subsection{Hàm ý chính sách}

Dựa vào kết quả thu được của nghiên cứu thực nghiệm này, tác giả gợi ý một số chính sách để thúc đẩy TKS giữa các nhân viên trong khu vực công. Vai trò quan trọng của $\mathrm{SC}$ và $\mathrm{OC}$ trong việc tăng cường TKS đã được phân tích và chứng minh ở nội dung bài báo này. Do đó, các nhà quản lý khu vực công nên nuôi dưỡng và phát triển $\mathrm{OC}$ vì những người đã cam kết với tổ chức có nhiều động lực tham gia vào TKS hơn. Để nhân viên có mức $\mathrm{OC}$ mạnh thì các lãnh đạo cần quan tâm đến các chiều kích then chốt của $\mathrm{SC}$, đó là sự tương tác giữa các $\mathrm{CBCCVC}$ ( $\mathrm{SSC}$ ), sự tin tưởng trong quan hệ với đồng nghiệp và tổ chức (RSC) cùng với mục tiêu, tầm nhìn chung (CSC). Để duy trì và nâng cao $\mathrm{SC}$ của nhân viên thì các tổ chức, các nhà quản lý nên kiến tạo bầu không khí thân mật cung cấp nhiều thời gian và không gian cho các hoạt động giao tiếp xã hội của $\mathrm{CBCCVC}$ bằng nhiều chương trình tương tác hơn liên quan đến cả cấp dưới và lãnh đạo cao nhất. Các chính sách, chương trình này nhằm giải đáp, chia sẻ các vấn đề không chỉ liên quan đến phúc lợi mà còn liên quan đến sự không hài lòng của CBCCVC trong thực hiện công việc. Việc tham gia vào các chương trình như vậy sẽ duy trì và nâng cao hơn nữa mối quan hệ giữa $\mathrm{CBCCVC}$ và lãnh đạo cũng như niềm tin vào tổ chức. Ngoài ra, các cuộc họp và các giao lưu chia sẻ tri thức không chính thức với sự tham gia của $\mathrm{CBCCVC}$ cũng có thể là nền tảng tốt để nhân viên đánh giá năng lực của đồng nghiệp và tăng cường sự tin tưởng lẫn nhau. Vai trò tích cực của tầm nhìn chung trong bối cảnh $\mathrm{OC}$ cho thấy mức độ cần thiết của việc định hình và truyền đạt rõ ràng những kỳ vọng về mục tiêu chung cho các CBCCVC trong cơ quan để khuyến khích thái độ và hành vi $\mathrm{OC}$ của nhân viên.

\section{Kết luận và hạn chế của nghiên cứu}

Nghiên cứu này đã phát triển và kiểm tra thực nghiệm mô hình mối quan hệ giữa $\mathrm{SC}, \mathrm{OC}$ và TKS trong khu vực công. Các minh chứng trong bối cảnh khu vực công đã bổ sung, làm giàu lý thuyết về $\mathrm{SC}$, bằng cách làm rõ vai trò của $\mathrm{SSC}, \mathrm{RSC}$ và $\mathrm{CSC}$ trong việc thúc đẩy $\mathrm{OC}$ để từ đó hình thành văn hóa TKS trong các tổ chức. Ngày nay, các tổ chức khu vực công muốn thành công dựa trên tri thức thì cần phải có các chiến lược quản lý tri thức phù hợp để làm giàu nguồn lực tri thức ẩn, mà theo bài báo này đó là các chính sách về $\mathrm{SC}$ và $\mathrm{OC}$ làm tiền đề cho các thực hành TKS hiệu quả.

Mặc dù, tác giả đã hoàn thành các mục tiêu ban đầu, nhưng vẫn còn một số hạn chế và cần tiếp tục nghiên cứu trong tương lai. Đầu tiên, nghiên cứu này chỉ xem xét các chiều kích của $\mathrm{SC}$ là tiền đề để thúc đẩy TKS thông qua $\mathrm{OC}$ nên tiếp theo cần khám phá thêm các nhân tố liên quan đến động lực cá nhân có ảnh hưởng đến $\mathrm{OC}$ không và mức độ như thế nào. Ngoài ra, nghiên cứu hiện tại chỉ xem $\mathrm{OC}$ là một biến tiềm ẩn nên các nghiên cứu sau có thể xem xét $\mathrm{OC}$ trong khuôn khổ 03 chiều kích khác nhau: quy tắc, tình cảm và lâu dài để phân tích sâu hơn. 


\section{Tài liệu tham khảo}

Allen, N. J., \& Meyer, J. P. (1990). The measurement and antecedents of affective, continuance, and normative commitment to the organization. Journal of Occupational Psychology, 63(1), 1-18.

Anderson, J. C., \& Gerbing, D. W. (1988). Structural equation modeling in practice: A review and recommended two-step approach. Psychological Bulletin, 103(3), 411-423.

Baker, W. E. (1990). Market networks and corporate behavior. American Journal of Sociology, 96(3), 589-625.

Bennet, D., \& Bennet, A. (2008). Engaging tacit knowledge in support of organizational learning. The Journal of Information and Knowledge Management System, 38(1), 72-94.

Bolino, M. C., Turnley, W. H., \& Bloodgood, J. M. (2002). Citizenship behavior and the creation of social capital in organizations. Academy of Management Review, 27(4), 505-522.

Borges, R., \& Mote, J. (2016). Is it the Brazilian way? Extending the comparison of public and private organizations. Public Organization Review, 16(4), 509-528.

Borges, R., Bernardi, M., \& Petrin, R. (2019). Cross-country findings on tacit knowledge sharing: Evidence from the Brazilian and Indonesian IT workers. Journal of Knowledge Management, 23(4), 742-762.

Bozionelos, N. (2008). Intra-organizational network resources: How they relate to career success and organizational commitment. Personnel Review, 37(3), 249-263.

Brockmann, E. N., \& Anthony, W. P. (1998). The influence of tacit knowledge and collective mind on strategic planning. Journal of Managerial Issues, 10(2), 204-222.

Chai, D. S., Hwang, S. J., \& Joo, B. K. (2017). Transformational leadership and organizational commitment in teams: The mediating roles of shared vision and team-goal commitment. Performance Improvement Quarterly, 30(2), 137-158.

Chiu, C. M., Hsu, M. H., \& Wang, E. T. (2006). Understanding knowledge sharing in virtual communities: An integration of social capital and social cognitive theories. Decision Support Systems, 42(3), 1872-1888.

Coetsee, L. (1999). From resistance to commitment. Public Administration Quarterly, 23(2), 204-222.

Coleman, J. S. (1988). Social capital in the creation of human capital. American Journal of Sociology, 94(Supplement), S95-S120.

Curado, C., \& Vieira, S. (2019). Trust, knowledge sharing and organizational commitment in SMEs. Personnel Review, 48(6), 1449-1468.

Fang, H., \& Zhang, S. (2014). A structural model of enterprise managers' tacit knowledge and personality traits. Social Behavior and Personalit, 42(5), 783-798.

Fornell, C., \& Larcker, D. F. (1981). Evaluating structural equation models with unobservable variables and measurement error. Journal of Marketing Research, 18(1), 39-50.

Gormley, D. K., \& Kennerly, S. (2010). Influence of work role and perceptions of climate on faculty organizational commitment. Journal of Professional Nursing, 26(2), 108-115.

Hair, J. F., Black, W. C., Babin, B. J., \& Anderson, R. E. (2014). Multivariate data analysis (7th ed.). London, UK: Pearson New International Edition.

Hau, Y. S., Kim, B., \& Lee, H. (2016). What drives employees to share their tacit knowledge in practice? Knowledge Management Research \& Practice, 14(3), 295-308. 
Herrgard, T. H. (2000). Difficulties in diffusion of tacit knowledge in organizations. Journal of Intellectual Capital, 1(4), 357-365.

Hislop, D. (2003). Linking human resource management and knowledge management via commitment: A review and research agenda. Employee Relations, 25(2), 182-202.

Holste, J. S., \& Fields, D. (2010). Trust and tacit knowledge sharing and use. Journal of Knowledge Management, 14(1), 128-140.

Hooff, B. V. D., \& Weenen, F. D. L. V. (2004). Committed to share: Commitment and CMC use as antecedents of knowledge sharing. Knowledge and Process Management, 11(1), 13-24.

Hsu, C. P., Chang, C. W., Huang, H. C., \& Chiang, C. Y. (2011). The relationships among social capital, organisational commitment and customer-oriented prosocial behaviour of hospital nurses. Journal of Clinical Nursing, 20(9/10), 1383-1392.

Jacobs, J. (1961). The death and life of great American cities. New York, NY: Random House.

Kline, R. B. (2015). Principles and practice of structural equation modeling (3rd ed.). NewYork, NY: The Guilford Press.

Laschinger, H. K. S., Finegan, J., \& Shamian, J. (2002). The impact of workplace empowerment, organizational trust on staff nurses' work satisfaction and organizational commitment. Advances in Health Care Management, 3(3), 59-85.

Lievens, F., \& Corte, W. D. (2008). Development and test of a model of external organizational commitment in human resources outsourcing. Human Resource Management, 47(3), 559579.

Lin, C. P. (2007). To share or not to share: Modeling tacit knowledge sharing, its mediators and antecedents. Journal of Business Ethics, 70(4), 411-428.

Manaf, H. A., Armstrong, S. J., Lawton, A., \& Harvey, W. S. (2018). Managerial tacit knowledge, individual performance, and the moderating role of employee personality. International Journal of Public Administration, 41(15), 1258-1270.

Meyer, J. P., \& Allen, N. J. (1991). A three-component conceptualization of organizational commitment. Human Resource Management Review, 1(1), 61-89.

Meyer, J. P., \& Allen, N. J. (1997). Commitment in the workplace: Theory, research, and application. Thousand Oaks, CA: Sage Publications.

Meyer, J. P., \& Herscovitch, L. (2001). Commitment in the workplace: Toward a general model. Human Resource Management Review, 11(3), 299-326.

Mohammed, N., \& Kamalanabhan, T. J. (2019). Tacit knowledge seeking from teammates: Unravelling the role of social capital. International Journal of Organizational Analysis, 28(3), 765-790.

Nahapiet, J., \& Ghoshal, S. (1998). Social capital, intellectual capital, and the organizational advantage. Academy of Management Review, 23(2), 242-266.

Nambudiri, R. (2012). Propensity to trust and organizational commitment: A study in the Indian pharmaceutical sector. The International Journal of Human Resource Management, 23(5), 977-986.

Nonaka, I. (1994). A dynamic theory of organizational knowledge creation. Organization Science, 5(1), 14-37.

Nonaka, I., \& Krogh, G. V. (2009). Perspective-tacit knowledge and knowledge conversion: Controversy and advancement in organizational knowledge creation theory. Organization 
Science, 20(3), 635-652.

Nonaka, I., \& Takeuchi, H. (1995). The knowledge creating company: How Japanese companies create the dynamics of innovation. New York, NY: Oxford University Press.

Nonaka, I., \& Toyama, R. (2003). The knowledge-creating theory revisited: Knowledge creation as a synthesizing process. Knowledge Management Research and Practice, 1(1), 2-10.

O'Reilly, C. A., \& Chatman, J. (1986). Organizational commitment and psychological attachment: The effects of compliance, identification, and internalization on prosocial behavior. Journal of Applied Psychology, 71(3), 492-499.

Porter, L. W., Steers, R. M., Mowday, R. T., \& Boulian, P. V. (1974). Organizational commitment, job satisfaction, and turnover among psychiatric technicians. Journal of Applied Psychology, 59(5), 603-609.

Putnam, R. D. (1995). Bowling alone: America's declining social capital. Journal of Democracy, 6(1), 65-78.

Putnam, R. D. (2000). Bowling alone: America's declining social capital. In L. Crothers \& C. Lockhart (Eds.), Culture and politics (pp. 223-234). New York, NY: Palgrave Macmillan.

Rasdi, R. M., \& Tangaraja, G. (2020). Knowledge-sharing behaviour in public service organisations: Determinants and the roles of affective commitment and normative commitment. European Journal of Training and Development. doi:10.1108/EJTD-022020-0028

Reed, K. K., Srinivasan, N., \& Doty, D. H. (2009). Adapting human and social capital to impact performance: Some empirical findings from the U.S. Personal Banking Sector. Journal of Managerial Issues, 21(1), 36-57.

Seibert, S. E., Kraimer, M. L., \& Liden, R. C. (2001). A social capital theory of career success. Academy of Management Journal, 44(2), 219-237.

Song, J. H., Kim, H. M., \& Kolb, J. A. (2009). The effect of learning organization culture on the relationship between interpersonal trust and organizational commitment. Human Resource Development Quarterly, 20(2), 147-167.

Tekingündüz, S., Top, M., Tengilimoğlu, D., \& Karabulut, E. (2017). Effect of organisational trust, job satisfaction, individual variables on the organisational commitment in healthcare services. Total Quality Management \& Business Excellence, 28(5/6), 522-541.

Tsai, W., \& Ghoshal, S. (1998). Social capital and value creation: The role of intrafirm networks. Academy of Management Journal, 41(4), 464-476.

Wagner, R. K., \& Sternberg, R. J. (1985). Practical intelligence in real-world pursuits: The role of tacit knowledge. Journal of Personality and Social Psychology, 49(2), 436-458.

Wang, Z., \& Wang, N. (2012). Knowledge sharing, innovation and firm performance. Expert Systems with Applications, 39(10), 8899-8908.

Watson, G. W., \& Papamarcos, S. D. (2002). Social capital and organizational commitment. Journal of Business and Psychology, 16(4), 537-552.

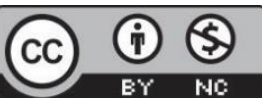

Creative Commons Attribution-NonCommercial 4.0 International License. 